\title{
Overexpression of ILK promotes temozolomide resistance in glioma cells
}

\author{
FENG LIANG $^{1}$, BING WANG ${ }^{1}$, LONG BAO ${ }^{1}$, YIN-SHENG ZHAO ${ }^{1}$, SHI-MIN ZHANG ${ }^{1}$ and SHU-QIN ZHANG ${ }^{2}$ \\ Departments of ${ }^{1}$ Neurosurgery and ${ }^{2}$ Pediatrics, The First Affiliated Hospital of Jinzhou Medical University, \\ Jinzhou, Liaoning 121001, P.R. China
}

Received December 11, 2015; Accepted November 29, 2016

DOI: $10.3892 / \mathrm{mmr} .2017 .6157$

\begin{abstract}
The present study aimed to investigate whether overexpression of integrin-linked kinase (ILK) affects drug resistance to temozolomide (TMZ) in glioma cells. To do this, a plasmid containing the ILK gene was transfected into the SHG-44 human glioma cell line, and cells were subsequently cultured in the absence or presence of TMZ. The expression levels of ILK, multidrug resistance-associated protein (MRP) and multi-drug resistance protein (MDR) were assessed in these cells. Cell growth and apoptosis were measured by MTT and Hoechst staining, and flow cytometry, respectively. In addition, the expression levels of B-cell lymphoma 2 (Bcl-2) and $\mathrm{Bcl}-2$-associated $\mathrm{x}$ protein (Bax), and caspase-3 activity, were evaluated. The ILK-overexpressing SHG-44 cell was successfully constructed, and demonstrated increased expression levels of ILK, MDR and MRP compared with untransfected cells. Cell growth in the ILK+TMZ group was significantly greater, and the percentage of apoptotic cells in the ILK+TMZ group was significantly reduced, compared with the $\mathrm{p}$ enhanced green fluorescent protein (EGFP)-C1+ TMZ empty vector control group. Expression levels of the anti-apoptotic protein Bcl-2 were significantly increased and those of the pro-apoptotic protein Bax were significantly decreased $(\mathrm{P}<0.01)$ in the ILK+TMZ group compared with the pEGFP-C1+TMZ group. In addition, the activity of caspase-3 in ILK+TMZ group was significantly decreased compared with the pEGFP-C1+TMZ group $(\mathrm{P}<0.01)$. Overexpression of ILK therefore promoted the proliferation of SHG-44 human glioma cells, reduced apoptosis and reduced sensitivity to TMZ via decreasing the activity of caspase-3.
\end{abstract}

Correspondence to: Dr Shu-Qin Zhang, Department of Pediatrics, The First Affiliated Hospital of Jinzhou Medical University, 5 Renmin Road, Jinzhou, Liaoning 121001, P.R. China

E-mail: shuqinzhang335@163.com

Key words: integrin-linked kinase, glioma cells, temozolomide, B-cell lymphoma 2, B-cell lymphoma 2-associated X protein, caspase-3

\section{Introduction}

As the most common primary tumor of the central nervous system, glioma accounts for $46 \%$ of all intracranial tumors (1-3). The incidence of cerebral glioma is 3-10 in 100,000 , comprising $1-3 \%$ of systemic malignant tumors. The median survival time of glioma patients who undergo surgery plus radiation and chemotherapy is only 8-11 months (4). Drug resistance to chemotherapy contributes to this poor prognosis (5); therefore, it is important to identify the mechanisms underlying drug resistance.

Integrin-linked kinase (ILK) was identified by Hannigan et al (6) using yeast two-hybrid screening. ILK cDNA is $1.8 \mathrm{~kb}$ long and encodes 452 amino acid residues, containing 3 structural units of Ser/Thr. ILK is normally expressed at low levels; ILK is activated upon adherence of extracellular matrix (ECM) to cells or by growth factors via the phosphoinositide 3-kinase (PI3K)-dependent signaling pathway. A previous study demonstrated that ILK overexpression in cells resulted in the activation of numerous signal transduction pathways (7). Overexpression of ILK in epithelial cells may lead to increased phosphorylation of protein kinase B (Akt) and glycogen synthase kinase 3 (8).

Previous studies have revealed that ILK may induce epithelial-mesenchymal transition, and promote the development of malignant tumor cells in bladder and pancreatic cancer (9-12). In addition, research performed by Duxbury et al (13) suggested that inhibition of the expression of ILK through RNA interference may enhance the sensitivity of pancreatic cancer cells to gemcitabine chemotherapy, This indicated that ILK expression may be associated with drug resistance of tumor cells. Overexpression of ILK has been detected in numerous human malignant tumors, and this over expression has been associated with poor prognosis (14-18). Few studies have investigated the association between ILK and drug resistance in glioma. The present study therefore investigated whether overexpression of ILK affected drug resistance to temozolomide in glioma cells.

\section{Materials and methods}

Cell line selection. U87 MG (Cell Bank of Type Culture Collection, Chinese Academy of Sciences, Shanghai, China), U373 (Shanghai Bioleaf Biotech Co., Ltd., Shanghai, China), U251 (Cell Bank of Type Culture Collection, Chinese 
Academy of Sciences), SHG-44 (Cell Bank of Type Culture Collection, Chinese Academy of Sciences) and T98 G (Co Bioer Biosciences Co., Ltd., Nanjing, China) human glioblastoma cell lines were cultured in RPMI-1640 medium (Gibco; Thermo Fisher Scientific, Inc., Waltham, MA, USA) containing $10 \%$ fetal bovine serum (FBS; Gibco; Thermo Fisher Scientific, Inc.) at $37^{\circ} \mathrm{C}$ and $5 \% \mathrm{CO}_{2}$. These cell lines were used for analysis of ILK protein expression levels by western blotting, to select a cell line with low expression levels for subsequent experiments.

Western blotting. Total proteins were extracted from cells using NP-40 (Beyotime Institute of Biotechnology, Shanghai, China) and the protein concentration was determined using the Bicinchoninic Acid assay. Total proteins $(50 \mu \mathrm{g})$ were subjected to $12 \%$ SDS-PAGE and transferred to polyvinylidene difluoride membranes (EMD Millipore, Billerica, MA, USA). Membranes were blocked with 5\% skimmed milk for $1 \mathrm{~h}$, and subsequently incubated with the following primary antibodies at $4^{\circ} \mathrm{C}$ overnight: Goat anti-ILK (1:5,000; clone, C-19; catalog no. sc-7516; Santa Cruz Biotechnology, Inc., Dallas, TX, USA), anti-multidrug resistance-associated protein (MRP; 1:5,000; cat. no. BA3340-2; rabbit anti human; Wuhan Boster Biological Technology, Ltd., Wuhan, China), anti-multidrug resistance protein (MDR; 1:1,000; cat. no. PB0162; rabbit anti human; Wuhan Boster Biological Technology, Ltd.), anti-B-cell lymphoma 2 (Bcl-2; 1:5,000; cat. no. BA0412; rabbit anti human; Wuhan Boster Biological Technology, Ltd) and anti-Bcl-2-associated X protein (Bax; 1:1,000; mouse anti human; cat. no. BA0315-2; Wuhan Boster Biological Technology, Ltd.). Membranes were washed three times with TBS and incubated with agoat anti rabbit, IgG-horseradish peroxidase (HRP; 1:5,000; cat. no. BA1054; Wuhan Boster Biological Technology, Ltd.) and goat anti mouse, IgG-HRP (1:5,000; cat. no. BA1050; Wuhan Boster Biological Technology, Ltd.) at $37^{\circ} \mathrm{C}$ for $45 \mathrm{~min}$. The proteins were visualized by RapidStep ${ }^{\mathrm{TM}}$ enhanced chemiluminescence reagent (cat. no. 345818-100ML; Merck Millipore). The Odyssey ${ }^{\circledR}$ Imaging system (LI-COR Biosciences, Lincoln, NE, USA) was used for semi-quantitative analysis (Quantity One image analysis system (BioRad Laboratories, Inc., Hercules, CA, USA). $\beta$-actin (1:10,000; cat. no. BM0626; Wuhan Boster Biological Technology, Ltd.) served as the control.

Establishment of ILK stable transfected cell line. The commercial vector is the ILK Human cDNA ORF clone (NM_004517), which was purchased from OriGene Technologies, Inc. (Rockville, MD, USA; cat. no. RC20154L1). The primer sequences are as follows: Forward, 5'-AAGGTACTCGAG CTATGGACGACATTTTCACTC-3' and reverse, 5'-ATC CAAGAATTCTCTACTTGTCCTGCATCTTCT-3'. PCR was performed in a Mx3000 machine using the following thermocycling conditions: An initial denaturation step at $95^{\circ} \mathrm{C}$ for $5 \mathrm{~min}$, followed by 30 cycles of $95^{\circ} \mathrm{C}$ for $30 \mathrm{sec}$ and $55^{\circ} \mathrm{C}$ for $30 \mathrm{sec}$, and a final step of $72^{\circ} \mathrm{C}$ for $90 \mathrm{sec}$ and $4^{\circ} \mathrm{C}$ for $5 \mathrm{~min}$. The amplified ILK gene was separated and detected by $1 \%$ agarose gel electrophoresis. The target DNA fragment was purified and obtained using a DNA Recovery kit (catalog no. DP1702; BioTeke Corporation, Beijing, China).
TA cloning. The obtained ILK gene was inserted into thepUM-T vector (BioTeke Corporation). Briefly, $0.3 \mathrm{pmol}$ ILK DNA was added to $1 \mu \mathrm{lpUM-T}$ vector $(0.03 \mathrm{pmol})$, and double-distilled $\mathrm{H}_{2} \mathrm{O}$ was added to a final volume of $5 \mu \mathrm{l}$. Subsequently, $5 \mu$ Solution I (BioTeke Corporation; cat. no. DP6814) was added and incubated at $16^{\circ} \mathrm{C}$ for $30 \mathrm{~min}$. The mixed solution was added to $100 \mu 1 \mathrm{JM} 109$ competent cells ( $2 \times 10^{9}$ cell/ml; Sigma-Aldrich; Merck Millipore, Darmstadt, Germany), and cultured for $30 \mathrm{~min}$ on ice, followed by $42^{\circ} \mathrm{C}$ for $90 \mathrm{sec}$ and $2 \mathrm{~min}$ on ice. Super Optimal Broth culture medium (800 $\mu \mathrm{l}$; BioTeke Corporation) was added, and cells were cultured for $1 \mathrm{~h}$ at $37^{\circ} \mathrm{C}$ with oscillation. The cells were cultured on Fast-Media ${ }^{\circledR}$ Ampicillin Agar (InvivoGen, San Diego, CA, USA) plates containing X-gal and isopropyl $\beta$-D-1-thiogalactopyranoside. White colonies containing ILK were selected, the inserted ILK fragment was confirmed by PCR, and positive clones were selected and sequenced.

Plasmid extraction and genetic recombination. Targeted plasmids [T-ILK and $\mathrm{p}$ enhanced green fluorescent protein (EGFP)-C1 (Clontech Laboratories, Inc., Mountainview, CA, USA)] were extracted using a Plasmid DNA Maxi-Preparation kit (catalog no. DP1008, BioTeke Corporation) according to the manufacturer's protocol and plasmid concentration was determined by ultraviolet spectrophotometry. Double enzyme digestion for T-ILK and pEGFP-C1 was performed at designated cutting sites as described previously (19), using Fast Digest EcoRI and Fast Digest XholI restriction enzymes (Thermo Fisher Scientific, Inc.). Following conjugation and transfection, JM109 competent cells were cultured in kanamycin-containing agar plates, single clones containing pEGFP-C1-ILK were selected and the inserted fragment length was determined by PCR.

Recombined pEGFP-C1-ILK clones were verified by automated sequencing using an ABI Prism 3730 DNA Sequencer (Applied Biosystems; Thermo Fisher Scientific, Inc.) and transfected into glioma cells. Stably transfected cells were selected using geneticin. Transfection of the empty pEGFP-C1 plasmid served as a control.

Reverse transcription-PCR (RT-PCR) for ILK mRNA expression levels. Total RNA was extracted from transfected cells using TRIzol ${ }^{\circledR}$ reagent (Takara Biotechnology Co., Ltd., Dalian, China) according to the manufacturer's protocol. RNA quality was detected by ultraviolet spectrophotometry and agarose gel electrophoresis. Total RNA $(1 \mu \mathrm{g})$ was reverse-transcribed to cDNA using the Takara RNA PCR kit (Takara Bio. Inc., Dalian, China; cat. no. RR019), and the ILK gene was amplified by PCR (Takara PCR Amplification kit (cat. no. RO11; Takara Bio. Inc.) with $\beta$-actin serving as a control. The primer sequences were as follows: Forward, 5'-ATGGAACCCTGA ACAAACACT-3' and reverse, 5'-AGCACATTTGGATGC GAGAAA-3' for ILK; and forward, 5'-CTTAGTTGCGTT ACACCCTTTCTTG-3' and reverse, 5'-CTGTCACCTTCA CCGTTCCAGTTT-3' for $\beta$-actin. The thermocycling conditions were as follows: An initial denaturation step at $95^{\circ} \mathrm{C}$ for $5 \mathrm{~min}$, followed by 30 cycles of $95^{\circ} \mathrm{C}$ for $20 \mathrm{sec}$ and $60^{\circ} \mathrm{C}$ for $20 \mathrm{sec}$, and a final step at $72^{\circ} \mathrm{C}$ for $30 \mathrm{sec}$ and $4^{\circ} \mathrm{C}$ for $5 \mathrm{~min}$. The amplified products were separated by $1.5 \%$ agarose gel and imaged using the Odyssey ${ }^{\circledR}$ Imaging system (LI-COR Biosciences). 
MTT assay. Stably transfected (pEGFP-C1-ILK) cells were cultured in RPMI-1640 medium containing 10\% FBS, and seeded into 96-well plates at a density of $3 \times 10^{3}$ cells per well. TMZ (Sigma-Aldrich; Merck Millipore; dissolved in DMSO $(10 \mathrm{mg}$ in $0.5 \mathrm{ml}$ ) and then diluted to $10 \mathrm{umol} / \mathrm{l}$ prior to experiment) was subsequently added. Cells were divided into five groups (5 wells/group): pEGFP-C1-ILK, pEGFP-C1+0.1\% dimethyl sulfoxide (DMSO), pEGFP-C1+100 $\mu \mathrm{M}$ TMZ, pEGFP-C1-ILK+ 0.1\% DMSO, pEGFP-C1-ILK+100 $\mu \mathrm{M}$ TMZ. An MTT assay was performed at 12, 24, 48 and $72 \mathrm{~h}$ after treatment. The medium was removed from each well and $100 \mu$ l MTT $(0.2 \mathrm{mg} / \mathrm{ml}$ in PBS$)$ was added in the absence of light; formazan crystals were produced over a 4-h incubation period at $37^{\circ} \mathrm{C}$. To dissolve crystals, $200 \mu \mathrm{l}$ DMSO was added to each well and the optical density (OD) at a wavelength of $490 \mathrm{~nm}$ was measured on a Tecan Spectra Fluor spectrophotometer (Tecan, Männedorf, Switzerland).

Hoechst staining. Hoechst staining was performed on the SHG 44, pEGFP-C1+0.1\% DMSO, pEGFP-C1+100 $\mu \mathrm{M}$ TMZ, pEGFP-C1-ILK $+0.1 \%$ DMSO and pEGFP-C1-ILK $+100 \mu \mathrm{M}$ TMZ groups $72 \mathrm{~h}$ following treatment. Cells were fixed on glass slides and stained with Hoechst for $5 \mathrm{~min}$. Cells were subsequently washed twice with PBS, treated with fluorescence quenching liquid, coverslipped and immediately observed under a fluorescence microscope (magnification, $\mathrm{x} 400$ ). A total of 5 random fields of vision were selected, and, the number of normal cells in each were counted, allowing for the average number to be calculated. Staining results were analyzed (the nuclei were pale blue and uniformly arranged in normal cells) and then analyzed in conjunction with flow cytometry results.

Flow cytometry. Flow cytometry was performed in the SHG44, pEGFP-C1+0.1\% DMSO, pEGFP-C1+100 $\mu \mathrm{M}$ TMZ, pEGFP-C1-ILK $+0.1 \%$ DMSO and pEGFP-C1-ILK $+100 \mu \mathrm{M}$ TMZ groups. The cells were inoculated in a T 25 culture flask until the cells adhered to the wall, following which TMZ and DMSO were added. After $72 \mathrm{~h}$ of treatment, cells were washed twice with PBS, digested by trypsin and centrifuged at $800 \mathrm{x} g$, $24^{\circ} \mathrm{C}$ for $5 \mathrm{~min}$. All components of the Annexin V-FITC/PI apoptosis double staining kit; cat. no. LHK601-050; JiaMay Biotech, Ltd., Beijing, China) were added sequentially, before incubating at room temperature for $15 \mathrm{~min}$, and analyzed within $1 \mathrm{~h}$ by flow cytometer (FACSCalibur; cat. no. FACS101; BD Biosciences, Franklin Lakes, NJ, USA) using FlowJo software (version, 7.6.1; FlowJo, LLC, Ashland, OR, USA).

Measurement of caspase-3 activity. A Caspase-3 Detection kit (Beyotime Institute of Biotechnology) was used to detect the activity of caspase- 3 . The transfected cells were transferred to a 6-well plate at a density of $1 \times 10^{6} / \mathrm{ml}$, and LAMD3100 (25 $\left.\mu \mathrm{l}\right)$ was added when cell confluence reached 65\%. Following culture for $12 \mathrm{~h}$, cells were washed in PBS, homogenized, lysed by NP-40 (Beyotime Institute of Biotechnology) and incubated on ice. Ac-DEVD-AMC (5 $\mu \mathrm{l}$; AAT Bioquest, Inc., Sunnyvale, CA, USA) was added to a reaction solution containing $50 \mu 1$ reaction buffer and $50 \mu 1$ protein solution, and incubated at $37^{\circ} \mathrm{C}$ for $4 \mathrm{~h}$. The absorption was measured at a wavelength of $405 \mathrm{~nm}$ using an enzyme standard meter
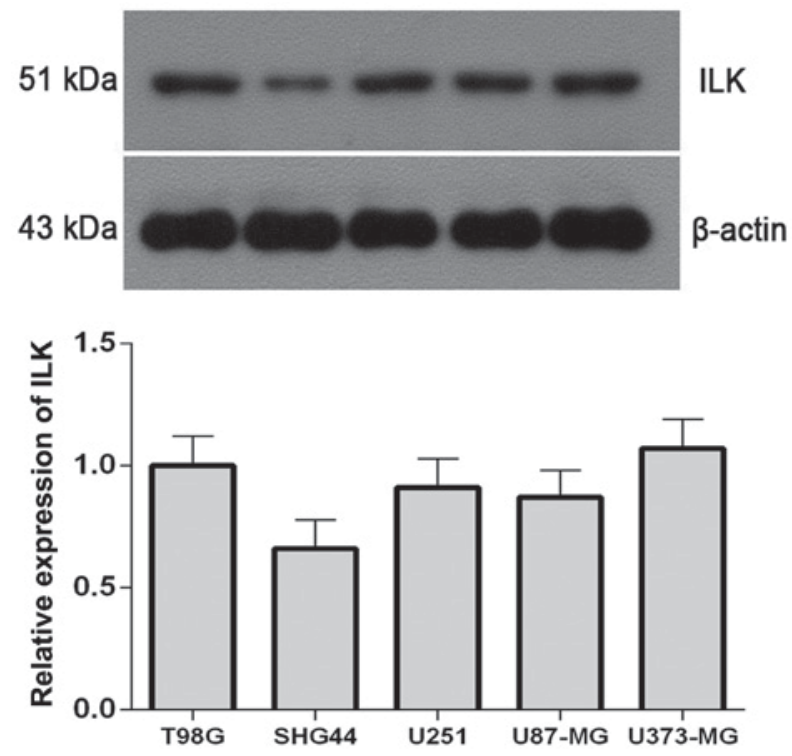

Figure 1. Western blot analysis of ILK protein expression levels in various glioma cell lines. Data are expressed as the mean \pm standard deviation. ILK, integrin-linked kinase.

(ELX-800; BiotekInstruments, Winooski, USA) andcaspase-3 activity was calculated using a standard curve.

Statistical analysis. SPSS software version 18.0 (SPSS, Inc., Chicago, IL, USA) was used to analyze the data. Data are presented as the mean \pm standard deviation. Analysis of variance analysis (along with Bonferroni adjustment as post-hoc analysis) and independent $t$-tests were performed to compare groups. $\mathrm{P}<0.05$ was considered to indicate a statistically significant difference.

\section{Results}

Cell selection. Western blot analysis revealed that the protein expression levels of ILK were lowest in the SHG-44 cell line (Fig. 1); therefore, subsequent experiments were performed on SHG-44 cells.

Amplification and transfection of ILK. The complete ILK gene fragment (length, $1.8 \mathrm{~kb}$ ) was amplified by PCR, and agarose gel electrophoresis results demonstrated that the size of the PCR product was consistent with the expected size of the ILK gene (Fig. 2A), indicating that the ILK gene was amplified.

The recombinant plasmid pEGFP-C1-ILK was identified by PCR, and the obtained PCR fragments were consistent with the expected size of $1.8 \mathrm{~kb}$ (Fig. 2B). In addition, analysis confirmed that the inserted fragment length presented no gene mutation in recombinant colonies (Fig. 2C). Furthermore, gene sequencing was performed to confirm the ILK gene segment in the ILK plasmid vector.

mRNA expression levels of ILK in SHG-44 transfected cells. RT-PCR results demonstrated that ILK mRNA expression levelsin stable transfected SHG-44 cellswere significantly greater compared with the controls (mock-transfected and empty vector-transfected SHG-44 cells; $\mathrm{P}=0.0067$; Fig. 3). 

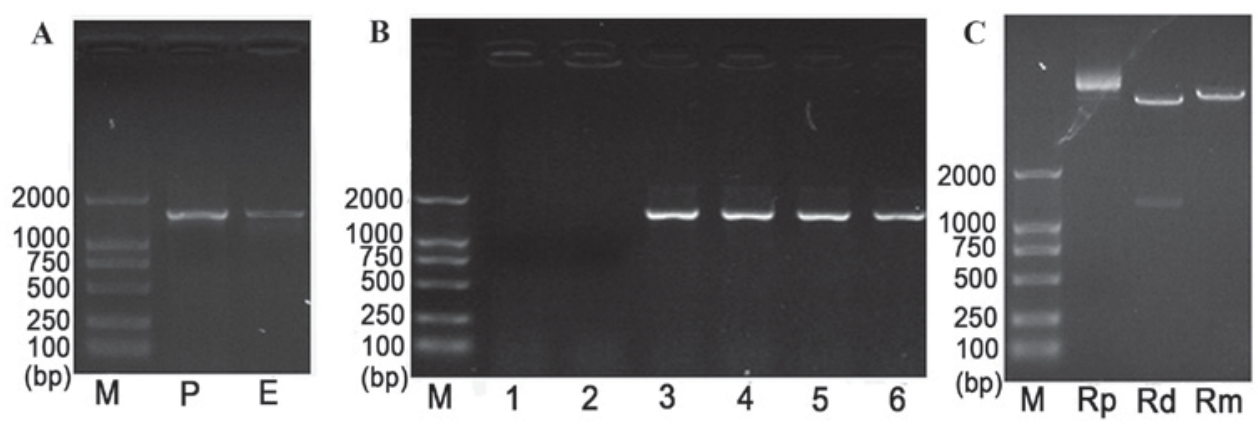

Figure 2. Identification of the ILK gene using PCR. PCR was performed on (A) purified ILK, (B) positive clones following transfection of ILK and (C) recombined T-ILK clone. M, marker; P, PCR product; E, enzyme-digested fragment; 1-6, selected positive clones; Rp, recombinant plasmid; Rd, double enzyme-digested recombinant plasmid; Rm, mono-enzyme-digested recombinant plasmid. ILK, integrin-linked kinase; PCR, polymerase chain reaction.
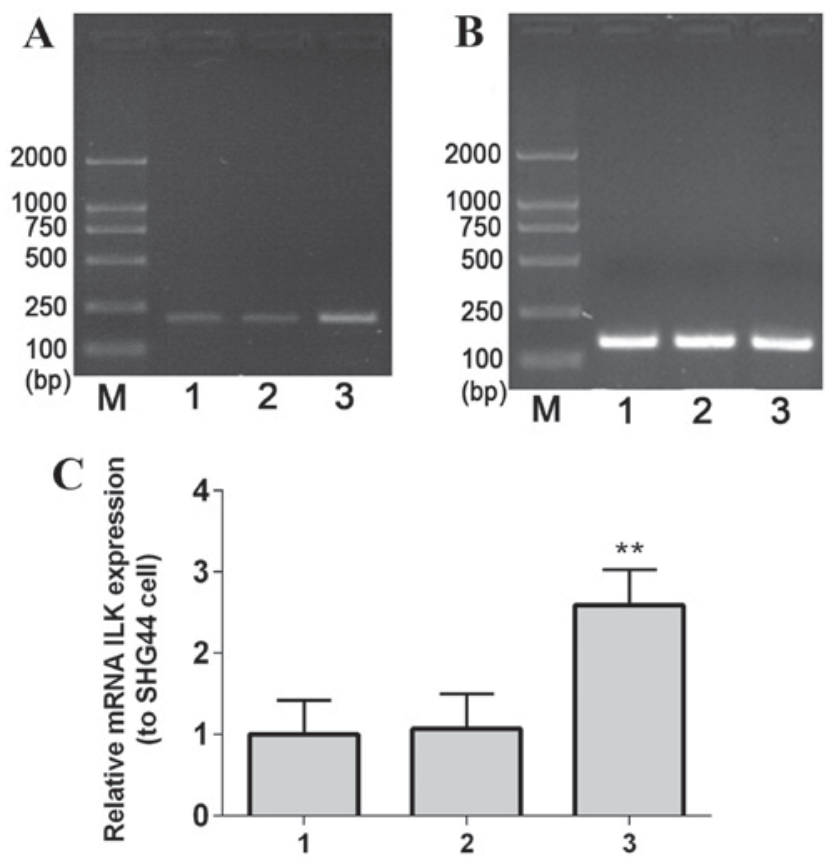

Figure 3. ILK mRNA expression levels in SHG-44 transfected cells, as determined by RT-PCR. (A) ILK and (B) $\beta$-actin mRNA expression levels. (C) Densitometry of the RT-PCR gels was performed to calculate the relative ILK mRNA expression levels. M, Marker; 1, SHG-44 cells; 2, SHG-44 cells transfected with empty vector; 3, SHG-44 cells stably-transfected with ILK. Data are expressed as the mean \pm standard deviation. ${ }^{* *} \mathrm{P}<0.01$ vs. SHG-44 cells. ILK, integrin-linked kinase; RT-PCR, reverse transcription-polymerase chain reaction.

Protein expression levels of ILK in SHG-44 transfected cells. Western blot analysis revealed that ILK protein expression levels were significantly increased in ILK stable transfected cells compared with controls (mock-transfected and empty vector-transfected SHG-44 cells; P=0.005; Fig. 4).

Protein expression levels of MDR and MRP. The protein expression levels of MDR (Fig. 5A) and MRP (Fig. 5B) were detected by western blot analysis. The protein expression levels of MDR and MRP in the stable transfected cells were significantly greater compared with the controls (mock-transfected and empty vector-transfected SHG-44 cells; $\mathrm{P}=0.0082$ ). This demonstrated that the transfected cells increase their resistance ability to TMZ through promoting the expression of MDR and MRP.
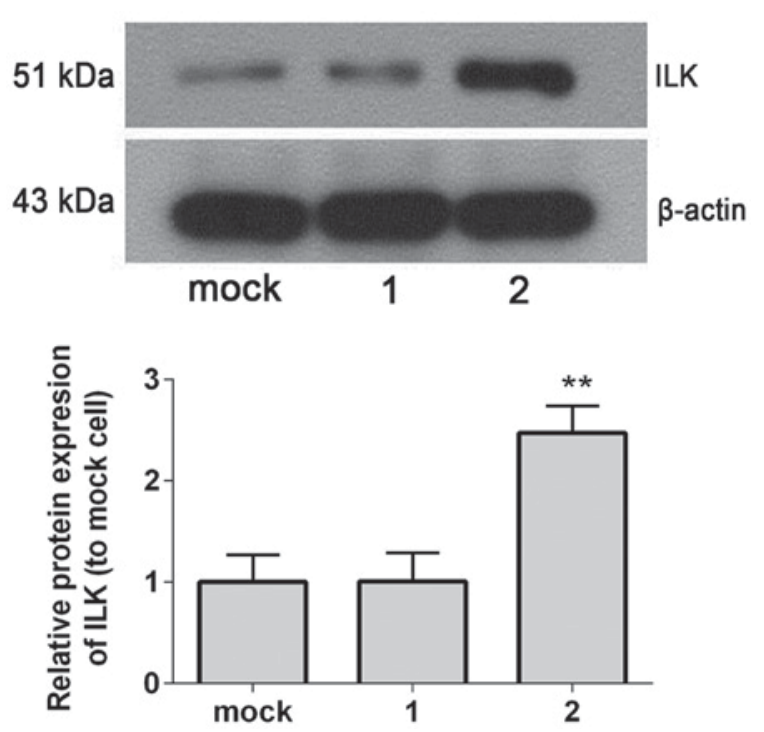

Figure 4. ILK protein expression levels in SHG-44 transfected cells, as determined by western blot analysis. Mock, SHG-44 cells; 2, SHG-44 cells transfected with empty vector; 3 , SHG-44 cells stably-transfected with ILK. Data are expressed as the mean \pm standard deviation. ${ }^{* *} \mathrm{P}<0.01$ vs. SHG-44 cells. ILK, integrin-linked kinase.

Cell growth measured by MTT. Cell proliferation was evaluated using an MTT assay; results are presented in Table I. In thepEGFP-C1-ILK transfected group (subsequently abbreviated to ILK), the OD values at 24,48 and $72 \mathrm{~h}$ following TMZ treatment group were significantly greater compared with the pEGFP-C1+TMZ group ( $\mathrm{P}=0.0074)$; at the same time points, the OD values in the ILK+DMSO group were increased compared with the pEGFP C1+DMSO group $(\mathrm{P}=0.0063)$. These results suggested that overexpression of ILK promotesSHG-44 human glioma cell proliferation.

Apoptosis of cells detected by Hoechst staining. Under a fluorescence microscope, apoptotic cells revealed nuclear dense staining or chunky dense staining; apoptosis occurred to the greatest extent in the pEGFP-C1+TMZ group. No significant differences were observed in the percentage of apoptosis between the SHG-44 and pEGFP-C1+DMSO groups; the percentage of apoptotic cells in the ILK+TMZ group was significantly reduced compared with the pEGFP-C1+TMZ group (Fig. 6). These results indicated that overexpression of 
Table I. Proliferation of cells

Time following treatment $(\mathrm{h})$.

\begin{tabular}{lccccc}
\cline { 2 - 6 } Group & \multicolumn{1}{c}{0} & \multicolumn{1}{c}{12} & \multicolumn{1}{c}{24} & \multicolumn{1}{c}{48} & 72 \\
\hline SHG-44 & $0.2290 \pm 0.0125$ & $0.3065 \pm 0.0164$ & $0.4448 \pm 0.0354$ & $0.5728 \pm 0.0342$ & $0.7953 \pm 0.0186$ \\
pEGFP-C1+DMSO & $0.2293 \pm 0.0144$ & $0.3083 \pm 0.0152$ & $0.4583 \pm 0.0295$ & $0.5723 \pm 0.0373$ & $0.8213 \pm 0.0867$ \\
pEGFP-C1+TMZ & $0.2235 \pm 0.0138$ & $0.3150 \pm 0.0274$ & $0.3720 \pm 0.0307$ & $0.4845 \pm 0.0456$ & $0.5798 \pm 0.0610$ \\
pEGFP-C1-ILK+ DMSO & $0.2303 \pm 0.0150$ & $0.4183 \pm 0.038$ & $0.7113 \pm 0.0480^{\mathrm{a}}$ & $1.1238 \pm 0.0885^{\mathrm{a}}$ & $1.3480 \pm 0.1284^{\mathrm{a}}$ \\
pEGFP-C1-ILK + TMZ & $0.2270 \pm 0.0138$ & $0.3360 \pm 0.0214$ & $0.5048 \pm 0.0128^{\mathrm{b}}$ & $0.8478 \pm 0.0836^{\mathrm{b}}$ & $1.0450 \pm 0.0910$
\end{tabular}

Optical density was measured at a wavelength of $490 \mathrm{~nm}$. Data are presented as the mean \pm standard deviation. ${ }^{\mathrm{a} P}<0.01 \mathrm{vs}$. pEGFP-C1+DMSO; ${ }^{\text {bP }}<0.01$ vs. pEGFP-C1+TMZ. EGFP, enhanced green fluorescent protein; DMSO, dimethyl sulfoxide; TMZ, temozolomide; ILK, integrin-linked kinase.

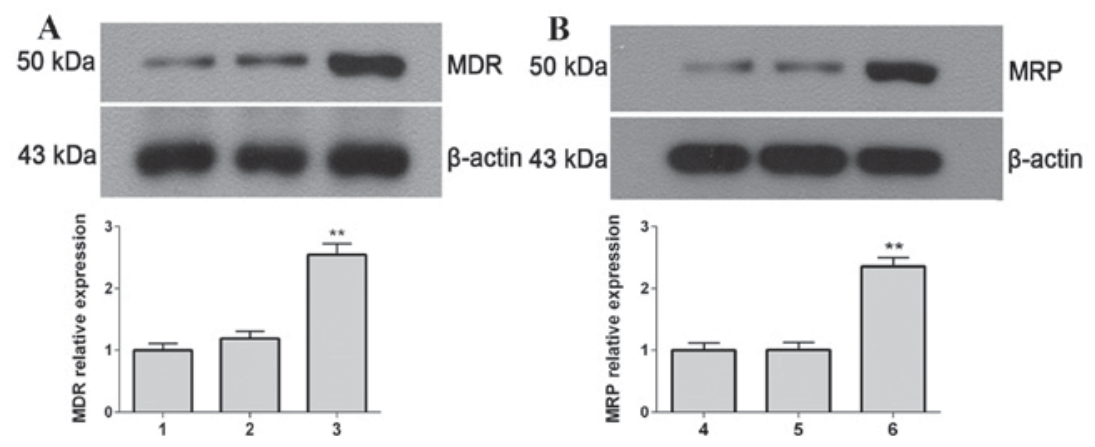

Figure 5. MDR and MRP protein expression levels in SHG-44 transfected cells, as determined by western blot analysis. (A) MDR and (B) MRP protein expression levels. 1, SHG-44 cells; 2, SHG-44 cells transfected with empty vector; 3, SHG-44 cell stably-transfected with ILK. Data are expressed as the mean \pm standard deviation. ${ }^{* *} \mathrm{P}<0.01$ vs. SHG-44 cells. MDR, multi-drug resistance protein; MRP, multidrug resistance-associated protein.

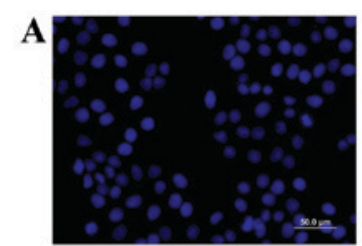

SHG44

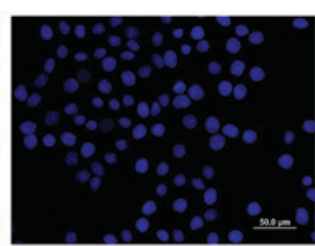

pEGFP-C1+DMSO

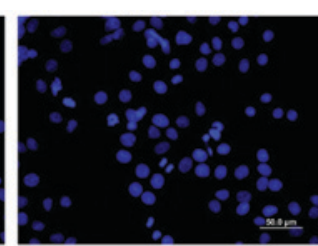

PEGFP-C1+TMZ

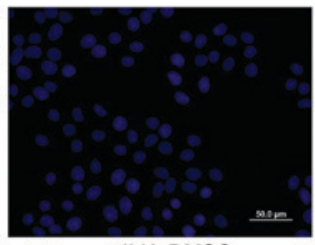

ILK+DMSO

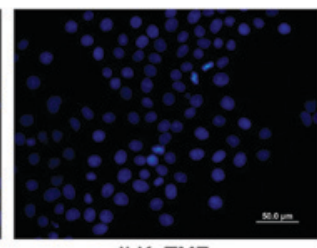

ILK+TMZ

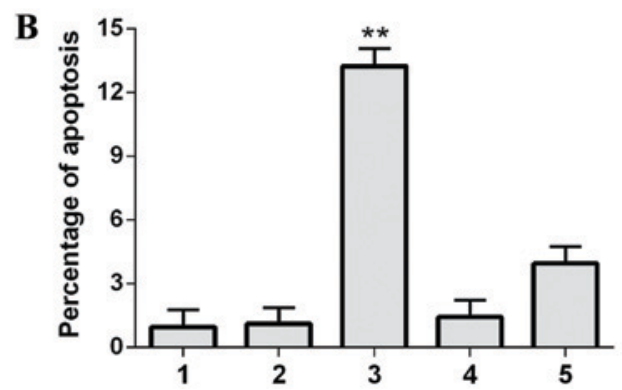

Figure 6. Apoptosis of SHG-44 transfected cells, as detected by Hoechst staining. (A) Representative images of Hoechst staining in the different groups Scale bar $=50 \mu \mathrm{m}$. (B) Quantification of Hoechst staining. 1, SHG-44 cells; 2, pEGFP-C1+DMSO; 3, pEGFP-C1+TMZ; 4, pEGFP-C1-ILK+DMSO; 5, pEGFP-C1-ILK+TMZ. Data are expressed as the mean \pm standard deviation. ${ }^{* *} \mathrm{P}<0.01$ vs. other groups. EGFP, enhanced green fluorescent protein; DMSO, dimethyl sulfoxide; TMZ, temozolomide; ILK, integrin-linked kinase. 

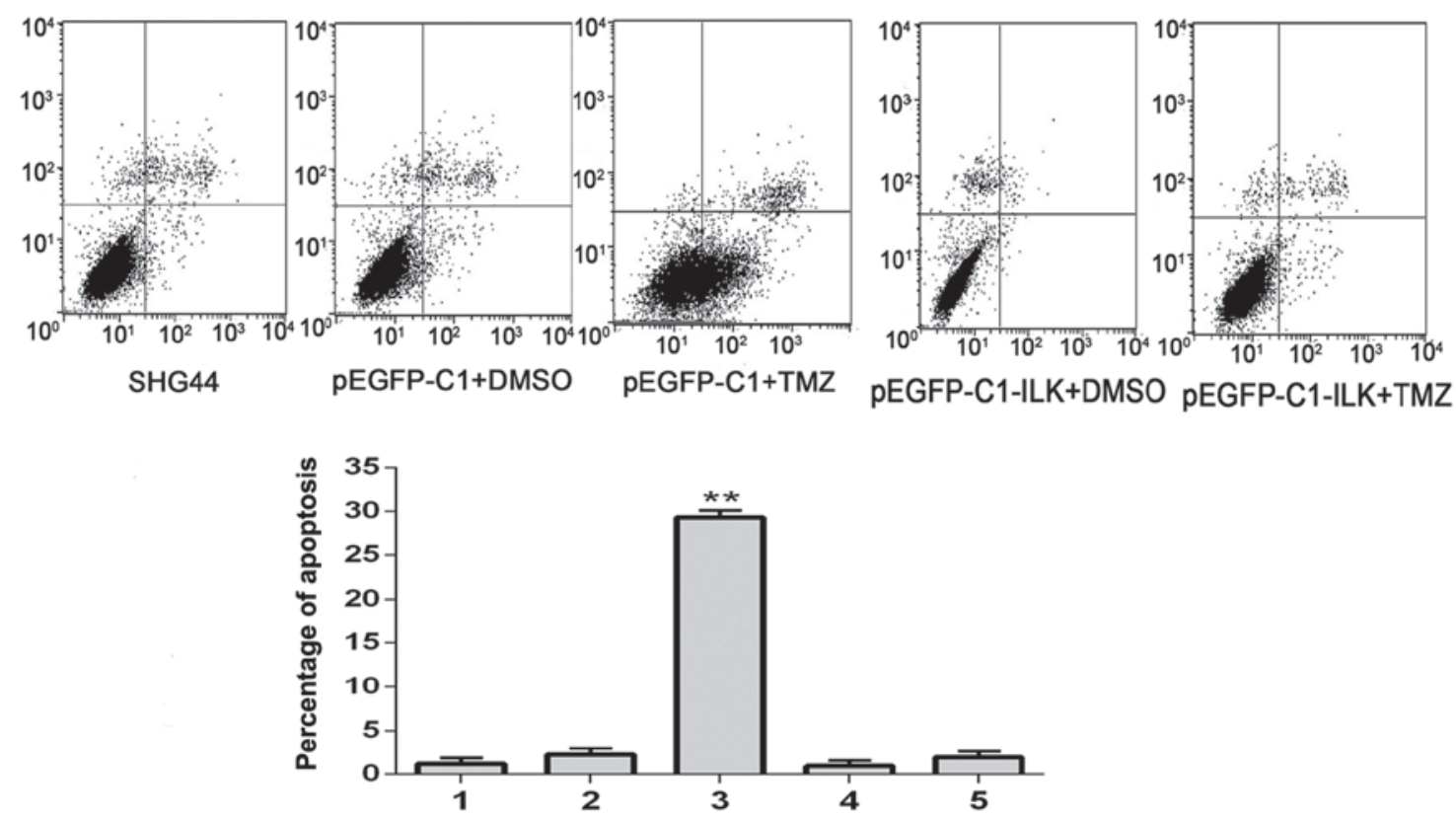

Figure 7. Early apoptosis of SHG-44 transfected cells, as detected by flow cytometric analysis. 1, SHG-44 cells; 2, pEGFP-C1+DMSO; 3, pEGFP-C1+TMZ; 4, pEGFP-C1-ILK+DMSO; 5, pEGFP-C1-ILK+TMZ. Data are expressed as the mean \pm standard deviation. ${ }^{* *} \mathrm{P}<0.01$ vs. other groups. EGFP, enhanced green fluorescent protein; DMSO, dimethyl sulfoxide; TMZ, temozolomide; ILK, integrin-linked kinase.
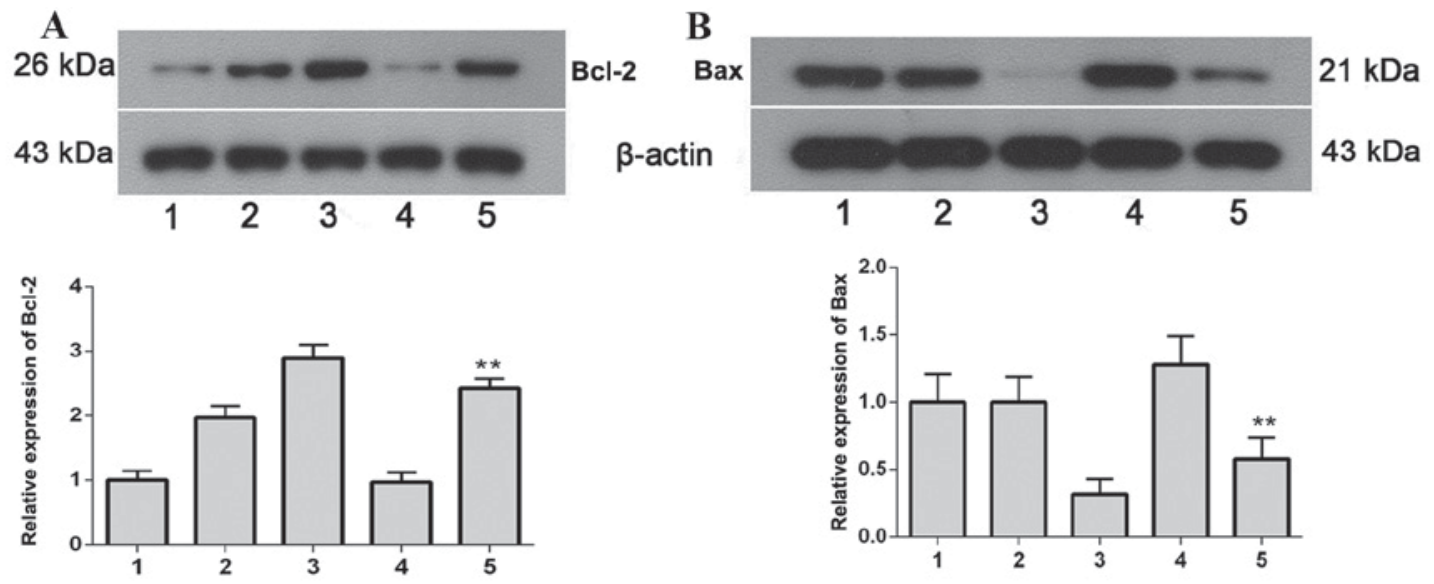

Figure 8. Bcl-2 and Bax protein expression levels in SHG-44 transfected cells, as determined by western blot analysis. (A) Bcl-2 and (B) Bax protein expression levels. 1, SHG-44 cells; 2, pEGFP-C1+DMSO; 3, pEGFP-C1-ILK+DMSO; 4, pEGFP-C1+TMZ; 5, pEGFP-C1-ILK+TMZ. Data are expressed as the mean \pm standard deviation. ${ }^{* *} \mathrm{P}<0.01$ vs. pEGFP-C1+TMZ. Bcl-2, B-cell lymphoma 2; Bax, B-cell lymphoma 2-associated X protein; EGFP, enhanced green fluorescent protein; DMSO, dimethyl sulfoxide; TMZ, temozolomide; ILK, integrin-linked kinase.

ILK reduced apoptosis in glioma cells, reducing their sensitivity to TMZ.

Flow cytometry. Flow cytometric analysis demonstrated that the percentage of early apoptotic cells (lower right quadrant) was greatest in the pEGFP-C1+TMZ group; the percentage of these cells was significantly reduced in the ILK+TMZ group $(\mathrm{P}=0.001$; Fig. 7$)$. These results suggested that the overexpression of ILK may decrease apoptosis in glioma cells treated with TMZ, and therefore decrease the sensitivity of cells to TMZ.

Protein expression levels of Bcl-2 and Bax. The protein expression levels of Bcl-2 (Fig. 8A) and Bax (Fig. 8B) were detected by western blot analysis. The protein expression levels of $\mathrm{Bcl}-2$, an anti-apoptotic protein, were significantly increased $(\mathrm{P}=0.0093)$ in the ILK+TMZ group compared with the pEGFP-C1+TMZ group; whereas the protein expression levels of the pro-apoptotic protein Bax were significantly decreased $(\mathrm{P}=0.0068)$ in the ILK+TMZ group compared with the pEGFP-C1+TMZ group. These results indicated that ILK increased expression of anti-apoptotic proteins, and decreased expression of pro-apoptotic proteins, thus reducing apoptosis in glioma cells and their sensitivity to TMZ.

Caspase-3 activity. The activity of caspase-3 in the ILK+TMZ group was significantly reduced compared with the pEGFP-C1+TMZ group ( $\mathrm{P}=0.0078$; Table II). These results suggested that ILK decreased the activity of caspase-3, thus decreasing apoptosis in glioma cells and their sensitivity to TMZ. 
Table II. Caspase-3 activity.

\begin{tabular}{lc}
\hline Group & $\begin{array}{c}\text { Caspase-3 activity } \\
(\mu \mathrm{M} / \text { gprotein })\end{array}$ \\
\hline SHG-44 & $17.77 \pm 0.89$ \\
pEGFP-C1+DMSO & $17.49 \pm 2.00$ \\
pEGFP-C1+TMZ & $24.19 \pm 3.13$ \\
pEGFP-C1-ILK+DMSO & $10.91 \pm 1.71^{\mathrm{a}}$ \\
pEGFP-C1-ILK+TMZ & $17.48 \pm 2.50$ \\
\hline
\end{tabular}

Data are presented as the mean \pm standard deviation. ${ }^{\mathrm{a}} \mathrm{P}<0.01$ pEGFP-C1-ILK+TMZ vs. pEGFP-C1+TMZ. EGFP, enhanced green fluorescent protein; DMSO, dimethyl sulfoxide; TMZ, temozolomide; ILK, integrin-linked kinase.

\section{Discussion}

The occurrence and development of tumors requires cells to perform various functions, including proliferation, independent growth, anchoring, apoptosis, angiogenesis, invasion and metastasis. ILK, a unique intracellular regulator, may contribute to these functions. In addition, ILK interacts with the protein kinase of cell adhesion receptors, as well as integrin and actin cytoskeleton growth factors. Evidence indicates that ILK may be a cancer gene, and that it may promote tumor cell survival or tumor progression by regulating various signaling pathways (20).

ILK serves a complex role in numerous cellular functions, including proliferation, motility, invasion, metastasis and angiogenesis $(21,22)$. A study by Lee et al (23) demonstrated that ILK regulates phosphorylation of Akt in phosphatase and tensin homolog deficient prostate cancer and breast cancer cells. Overexpression of ILK has been associated with tumor progression and poor prognosis in patients with rectal cancer (24). Monferran et al (25) revealed that the sensitivity of glioma cells to ionizing radiation was regulated by RhoB and ILK. Lanvin et al (26) demonstrated that ILK altered the radiation sensitivity of glioma via the regulation of the hypoxia-inducible factor $1 \alpha$ and survivin pathway and the mediation of mitochondrial death. These findings indicated that ILK may be a potential target for the treatment of glioma (20). The aim of the present study was to investigate biological behaviors, including proliferation and apoptosis, in ILK stable transfected SHG-44 cells in vitro, and investigate the sensitivity of transfected glioma cells to TMZ.

In the present study, western blot analysis and RT-PCR revealed that the expression levels of ILK protein and mRNA in the stable transfected ILK cell line was significantly greater compared with the mock- and empty vector-transfected groups, which indicated the successful construction of the ILK stable transfected cell line. The protein expression levels of MDR and MRP were significantly increased in ILK-transfected cells, which made their resistance ability to TMZ increased.

Bcl-2 family proteins are important regulatory factors in the endogenous mitochondrial-dependent apoptosis pathway. In the past 20 years, in vitro and in vivo studies have revealed that $\mathrm{Bcl}-2$ family proteins serve important roles in the drug resistance of tumor cells. The endogenous apoptosis pathway may be activated by numerous factors, including chemotherapy agents, growth deprivation, mitochondria membrane breakdown and apoptosis factors, particularly the release of cytochrome $\mathrm{C}$ from mitochondria into the cytoplasm (27). The mitochondrial apoptosis pathway is mediated by a complex network composed of pro- and anti-apoptotic proteins of the Bcl-2 family (28). Through upregulating anti-apoptotic proteins or downregulating pro-apoptotic proteins, the mitochondrial apoptotic pathway may determine whether gliomas are sensitive to radiation and chemotherapy (29). Western blot analysis demonstrated that ILK increased the expression of the anti-apoptotic protein Bcl-2 significantly and decreased the expression of the pro-apoptotic protein Baxin human glioma cells.

MTT assays revealed that following 24, 48 and $72 \mathrm{~h}$ of treatment with TMZ or DMSO, OD values in the ILK stable transfected group were significantly greater compared with the empty vector group, which indicated that the overexpression of ILK may promote the proliferation of TMZ-treated glioma cells. Hoechst staining revealed that the percentage of apoptotic cells in the ILK stable transfected group was significantly decreased compared with the empty vector group. Therefore, ILK may promote proliferation and decrease apoptosis in TMZ-treated cells.

Song et al (30) indicated that ILK influenced the MDR gene expression via the PI3K-Akt and mitogen-activated protein kinase-extracellular signal-regulated kinase signaling pathways, regulating the progress of gastric cancer. It is well known that caspase activation is a typical marker of early and late apoptosis. The activity of caspase- 3 is an important indicator to determine whether apoptosis is occurring in cells or tissues (31). Duxbury et al (13) demonstrated that ILK increased gemcitabine resistance in pancreatic cancer cells, and that knockdown of ILK increased the sensitivity of pancreatic cancer cells to gemcitabine via increasing caspase-3-mediated apoptosis. Therefore, ILK may regulate the sensitivity of glioma cells to TMZ. The results of the present study revealed that the activity of caspase-3 was significantly reduced in the ILK stable transfected cells compared with the empty vector group. The expression of ILK in glioma cells may decrease apoptosis in tumor cells via the downregulation of caspase-3 activity, thereby reducing the sensitivity of tumor cells to TMZ.

In conclusion, the results of the present study demonstrated that overexpression of ILK in SHG-44 glioma cells downregulated the expression of the anti-apoptotic protein Bcl-2, increased the expression of Bax, and decreased the activity of the key enzyme caspase-3, thus reducing the apoptosis of tumor cells, promoting their proliferation, and reducing the sensitivity of SHG-44 glioma cells to TMZ. The present results suggested that ILK may serve as a potential therapeutic target for glioma.

\section{Acknowledgements}

The present study was supported by the Doctor Startup Foundation in Liaoning Province (grant no. 201501101) and the Technology Startup Foundation of The First Affiliated Hospital of Liaoning Medical University (grant no. FYK201214). 


\section{References}

1. DeAngelis LM: Brain tumors. N Engl J Med 344: 114-123, 2001

2. Van Meir EG, Hadjipanayis CG, Norden AD, Shu HK, Wen PY and Olson JJ: Exciting new advances in neuro-oncology: The avenue to a cure for malignant glioma. CA Cancer J Clin 60 : 166-193, 2010.

3. Asklund T,Malmstrom A, Bergqvist M, Björ O and Henriksson R: Brain tumors in Sweden: Data from a population-based registry 1999-2012. Acta Oncol 54: 377-384, 2015.

4. Stupp R, Tonn JC, Brada M and Pentheroudakis G; ESMO Guidelines Working Group: High-grade malignant glioma: ESMO clinical practice guidelines for diagnosis, treatment and follow-up. Ann Oncol 21 (Suppl 5): v190-v193, 2010.

5. Mrugala MM, Adair J and Kiem HP: Temozolomide: Expanding its role in brain cancer. Drugs Today (Barc) 46: 833-846, 2010.

6. Hannigan GE, Leung-Hagesteijn C, Fitz-Gibbon L, Coppolino MG, Radeva G, Filmus J, Bell JC and Dedhar S: Regulation of cell adhesion and anchorage-dependent growth by a new beta 1-integrin-linked protein kinase. Nature 379: 91-96, 1996.

7. Dedhar S: Cell-substrate interactions and signaling through ILK. Curr Opin Cell Biol 12: 250-256, 2000.

8. Schmitz M, Grignard G, Margue C, Dippel W, Capesius C, Mossong J, Nathan M, Giacchi S, Scheiden R and Kieffer N: Complete loss of PTEN expression as a possible early prognostic marker for prostate cancer metastasis. Int J Cancer 120: 1284-1292, 2007.

9. Gil D, Ciołczyk-Wierzbicka D, Dulińska-Litewka J, Zwawa K, McCubrey JA and Laidler P: The mechanism of contribution of integrin linked kinase (ILK) to epithelial-mesenchymal transition (EMT). Adv Enzyme Regul 51: 195-207, 2011.

10. Matsui Y, Assi K, Ogawa O, Raven PA, Dedhar S, Gleave ME, Salh B and So AI: The importance of integrin-linked kinase in the regulation of bladder cancer invasion. Int J Cancer 130: 521-531, 2012

11. Serrano I, McDonald PC, Lock FE and Dedhar S: Role of the integrin-linked kinase (ILK)/Rictor complex in TGF $\beta$-1-induced epithelial-mesenchymal transition (EMT). Oncogene 32: 50-60, 2013.

12. Chen D, Zhang Y, Zhang X, Li J, Han B, Liu S, Wang L, Ling Y, Mao $S$ and Wang $X$ : Overexpression of integrin-linked kinase correlates with malignant phenotype in non-small cell lung cancer and promotes lung cancer cell invasion and migration via regulating epithelial-mesenchymal transition (EMT)-related genes. Acta Histochem 115: 128-136, 2013.

13. Duxbury MS, Ito H, Benoit E, Waseem T, Ashley SW and Whang EE: RNA interference demonstrates a novel role for integrin-linked kinase as a determinant of pancreatic adenocarcinoma cell gemcitabine chemoresistance. Clin Cancer Res 11: 3433-3438, 2005.

14. Dai DL, Makretsov N, Campos EI, Huang C, Zhou Y, Huntsman D, Martinka M and Li G: Increased expression of integrin-linked kinase is correlated with melanoma progression and poor patient survival. Clin Cancer Res 9: 4409-4414, 2003.

15. Sawai H, Okada Y, Funahashi H, Matsuo Y, Takahashi H, Takeyama $\mathrm{H}$ and Manabe T: Integrin-linked kinase activity is associated with interleukin-1 alpha-induced progressive behavior of pancreatic cancer and poor patient survival. Oncogene 25 : 3237-3246, 2006.
16. Li J, Zhang H, Wu J, Guan H, Yuan J, Huang Z and Li M: Prognostic significance of integrin-linked kinasel overexpression in astrocytoma. Int J Cancer 126: 1436-1444, 2010.

17. Yu J, Shi R, Zhang D, Wang E and Qiu X: Expression of integrin-linked kinase in lung squamous cell carcinoma and adenocarcinoma: Correlation with E-cadherin expression, tumor microvessel density and clinical outcome. Virchows Arch 458: 99-107, 2011.

18. Zhao D, Tang XF, Yang K, Liu JY and Ma XR: Over-expression of integrin-linked kinase correlates with aberrant expression of Snail, E-cadherin and N-cadherin in oral squamous cell carcinoma: Implications in tumor progression and metastasis. Clin Exp Metastasis 29: 957-969, 2012

19. Zhuang X, Lv M, Zhong Z, Zhang L, Jiang R and Chen J: Interplay between intergrin-linked kinase and ribonuclease inhibitor affects growth and metastasis of bladder cancer through signaling ILK pathways. J Exp Clin Cancer Res 35: 130, 2016.

20. Hannigan G, Troussard AA and Dedhar S: Integrin-linked kinase: A cancer therapeutic target unique among its ILK. Nat Rev Cancer 5: 51-63, 2005.

21. Persad S and Dedhar S: The role of integrin-linked kinase (ILK) in cancer progression. Cancer Metastasis Rev 22: 375-384, 2003

22. McDonald PC, Fielding AB and Dedhar S: Integrin-linked kinase-essential roles in physiology and cancer biology. J Cell Sci 121: 3121-3132, 2008.

23. Lee SL, Chou CC, Chuang HC, Hsu EC, Chiu PC, Kulp SK, Byrd JC and Chen CS: Functional role of mTORC2 versus integrin-linked kinase in mediating Ser473-Akt phosphorylation in PTEN-negative prostate and breast cancer cell lines. PLoS One 8: e67149, 2013

24. Li R, Liu B, Yin H, Sun W, Yin J and Su Q: Overexpression of integrin-linked kinase (ILK) is associated with tumor progression and an unfavorable prognosis in patients with colorectal cancer. J Mol Histol 44: 183-189, 2013.

25. Monferran S, Skuli N, Delmas C, Favre G, Bonnet J, Cohen-Jonathan-Moyal E and Toulas C: Alphavbeta3 and alphavbeta5 integrins control glioma cell response to ionising radiation through ILK and RhoB. Int J Cancer 123: 357-364, 2008.

26. Lanvin O, Monferran S, Delmas C, Couderc B, Toulas C and Cohen-Jonathan-Moyal E: Radiation-induced mitotic cell death and glioblastoma radioresistance: A new regulating pathway controlled by integrin-linked kinase, hypoxia-inducible factor 1 alpha and survivin in U87 cells. Eur J Cancer 49: 2884-2891, 2013.

27. Hotchkiss RS, Strasser A, McDunn JE and Swanson PE: Cell death. N Engl J Med 361: 1570-1583, 2009.

28. Zhang T and Saghatelian A: Emerging roles of lipids in BCL-2 family-regulated apoptosis. Biochim Biophys Acta 1831: 1542-1554, 2013

29. Kouri FM, Jensen SA and Stegh AH: The role of Bcl-2 family proteins in therapy responses of malignant astrocytic gliomas: Bcl2L12 and beyond. ScientificWorldJournal 2012: 838916 , 2012.

30. Song W, Jiang R and Zhao CM: Role of integrin-linked kinase in multi-drug resistance of human gastric carcinoma SGC7901/DDP cells. Asian Pac J Cancer Prev 13: 5619-5625, 2012.

31. Mazumder S, Plesca D and Almasan A: Caspase-3 activation is a critical determinant of genotoxic stress-induced apoptosis. Methods Mol Biol 414: 13-21, 2008. 